\title{
Haltungs- und fütterungsbedingte Erkrankungen der Streifenköpfigen Bartagame (Pogona vitticeps)
}

\author{
K. Alexandra Dörnath
}

Pogona vitticeps ist die mittlerweile in der Terraristik beliebteste Echse und wird daher in der Exotenpraxis sehr häufig vorgestellt. Für ihre optimale Gesundheit und artgerechte Haltung muss im Terrarium das Klima des Herkunftsgebietes künstlich erzeugt werden und das Nahrungsangebot muss dem des natürlichen Habitats zumindest ähneln. Bei Beachtung unten aufgeführter Haltungs- und Fütterungsempfehlungen sind diese ruhigen und zutraulichen Agamen relativ einfach zu halten. Allerdings muss der Halter bereit und in der Lage sein, Geld in Technik sowie laufende Unterhaltungskosten, insbesondere in Strom, zu investieren.

\section{Biologie}

Pogona vitticeps ist tagaktiv und kommt in busch- und baumbestandenen heißen, trockenen Arealen mit extrem heller Sonneneinstrahlung im östlichen zentralen Australien vor. Oft sonnen sich diese Agamen auf exponierten Plätzen, während sie ihr Territorium beobachten. In Menschenhand können sie über 9 Jahre alt werden. Die Geschlechtsreife ist - wie bei allen Reptilien - nicht vom Alter, sondern von der Größe abhängig. Das Weibchen kann mehrere Gelege im Jahr mit jeweils bis zu ca. 40 weichschaligen Eiern produzieren. Die Zeitigungsdauer beträgt bei $30^{\circ} \mathrm{C}$ ca. $60-70$, bei $26^{\circ} \mathrm{C}$ ca. 89-96 Tage. Inzwischen werden Farbmorphen wie „Sandfire“, „SandfireGold“ und „Pastels“ sowie schuppenlose Bartagamen („Silkback“, „Leatherback“) gezüchtet, die nicht in der Wildbahn vorkommen. Letztere sind allerdings anfällig für Sonnenbrand.

\section{Haltung}

\section{Gruppenzusammensetzung}

Gut eignet sich eine Gruppenzusammensetzung aus 1 Männchen („Bock“) mit 2-4 Weibchen ähnlicher Größe. Böcke untereinander sind unverträglich, während Weibchen meist zusammen gehalten werden können. Von paarweiser Haltung ist Abstand zu nehmen, denn insbesondere nach der Winterruhe wird das Weibchen beinahe dauerhaft vom Paarungsverhalten des Bockes gestresst. Eine Einzelhaltung von Bartagamen ist der in einer sozial unverträglichen Gruppe vorzuziehen.

\section{Terrarium}

Das Terrarium für ein Tier mit einer KopfRumpf-Länge von ca. 25 cm sollte in etwa folgende Maße haben:

- Länge: 120-150 cm

- Tiefe: $50-60 \mathrm{~cm}$

- Höhe: 70-80 cm

Es eignen sich Terrarien, die nur von der Frontseite aus einsichtig sind. Die dreidimensionale Gestaltung der Rück- und Seitenwände kann mittels Styropor und Fliesenkleber, in den Sand eingestreut wird, erfolgen. Das Verkleben der Wände muss großflächig, nicht punktuell, mittels Aquariensilikon erfolgen, denn sonst geraten Futtertiere hinter die Wände und vermehren sich dort.

\section{Beleuchtung und Temperatur}

Die Beleuchtungsdauer hat sich am jahreszeitlichen Verlauf der Sonneneinstrahlung des Ursprungshabitats zu orientieren. So sollte diese im Sommer mindestens 12-14, im Winter 4-7 Stunden betragen. Für die Erzeugung der Grundhelligkeit eignen sich T5-Tageslichtröhren, als künstliche Sonne hochwertige Halogenmetalldampflampen wie die Bright Sun UV Desert (Lucky Reptile) oder Quecksilberdampflampen wie die PowerSun (Zoo Med). Diese Metalldampflampen erzeugen lebensnotwendige Wärme und Helligkeit sowie für die psychische Gesundheit wichtige UV-Aund zum Schutz vor Knochenweiche essenzielle UV-B-Strahlung. Der Mindestabstand zum Tier ist unbedingt einzuhalten, denn sonst können Sonnenbrand, Hautkrebs und Netzhautprobleme auftreten.

Als wechselwarmes Tier benötigt die Bartagame einen Temperaturgradienten, der im Sommer zwischen $28^{\circ} \mathrm{C}$ und $55^{\circ} \mathrm{C}$ liegen muss. Mögliche Optionen zur technischen Steuerung eines naturidentischen Artenbeckens sind Sonnenaufgangs- und Sonnenuntergangssimulationen, Thermo- und Hygro-Kontrollelemente sowie eine dem Jahresverlauf entsprechende programmierte Tageslängensteuerung (z.B. Season Control, http://www.seasoncontrol.eu).

\section{Bodengrund}

Der mindestens 10-15 cm tiefe Bodengrund sollte größtenteils aus einer Mischung von Terrariensand mit Ton oder Lehm, die mit Wasser vermengt wird, bestehen. Ein weiterer Bereich kann mit losem, kalziumangereichertem Sand, ein anderer mit Rindenmulch, Erde sowie Moos gefüllt werden. So entsteht im Terrarium neben einem Temperatur- auch ein Luftfeuchtigkeitsgradient. Eine kostengünstige Variante zu Terrariensand stellt Flusssand dar. Weder staubiger Vogelsand noch scharfkantiger Spielkastensand sollte zum Einsatz kommen.

\section{Strukturierung des Terrariums}

Bei der Haltung von Weibchen, auch ohne Bock, muss eine adäquate Eiablagestelle angeboten werden. Wichtiges Inventar sind Höhlen (z. B. Korkröhren, Blumentöpfe), Klettermöglichkeiten (model- 
lierte Rückwände, Äste u.a.) sowie Steine und Schieferplatten.

Bartagamen - wie alle Reptilien - sollten sich keinesfalls außerhalb des Terrariums aufhalten, denn dort gibt es keine Zonen mit optimaler Vorzugstemperatur und es bestehen Unfallgefahren für die Tiere. Zudem ist eine Haltung außerhalb des Beckens unhygienisch, denn die Tiere werden nicht stubenrein.

$\mathrm{Zu}$ einer artgerechten Haltung gehört eine ca. 6- bis 8-wöchige Kühlphase für alle Altersklassen, die das Immunsystem stärkt, die Lebensdauer verlängert und die geschlechtsreifen Bartagamen paarungsbereit macht. Während dieser Ruhephase sollten die Reptilien keinesfalls gestört werden.

\section{Fütterung}

Eine artgerechte Fütterung orientiert sich am Futtervorkommen im natürlichen Habitat. Pogona vitticeps ernährt sich herbivor und insektivor.

Den Tieren sind täglich Kräuter und Blüten, am besten in getrockneter Form, anzubieten. Für den menschlichen Verzehr kultiviertes Gemüse, Obst oder angebauter Salat sind keine artgerechte Nahrung für Bartagamen, denn diese sind zu rohfaserarm und Obst zudem zu zuckerreich - schließlich leben diese Tiere weder in Obst- noch Gemüseplantagen oder in Salatfeldern.
Die Insekten sollten vor ihrer Verfütterung artspezifisch gefüttert und nur restriktiv angeboten werden, um einer Verfettung sowie zu schnellem Wachstum („Dampfaufzucht“) der Bartagame vorzubeugen. Dabei sollten:

- Jungtiere maximal 2-mal pro Woche

- Adulte höchstens 1-mal pro Woche mit Insekten gefüttert werden. Die Futtertiere sollten nicht größer als das Maul der Bartagame sein.

Das Angebot an Kräutern, Blüten sowie Insekten ist möglichst abwechslungsreich zu gestalten. Auf Mehlwürmer („Fastfood“ für Bartagamen) sollte unbedingt verzichtet werden. Kalzium, z.B. in Form geraspelter und zerstoßener Sepia oder Mineralsteine, muss ad libitum zur Verfügung stehen. Es darf nicht mit dem Futter verabreicht werden, denn die Tiere können durch eine übermäßige zwangsweise Aufnahme an einer Pseudogicht erkranken. Für das Bestäuben der Insekten eignet sich Korvimin ${ }^{\circledR}$ ZVT + Reptil (WDT) ausgezeichnet. Obwohl Bartagamen aus Trockengebieten stammen, sollte ihnen stets frisches und sauberes Wasser zur Verfügung stehen.

\section{Die Streifenköpfige Bartagame als Patient}

Der Transport der Bartagame in die Tierarztpraxis muss in einem thermostabilen, temperaturkontrollierten und blickdichten Behältnis (Styroporbox, Kühltasche) bei ca. $28-30^{\circ} \mathrm{C}$ erfolgen. Jedes
Tier sollte zur Stressminimierung separat transportiert werden. Auf Luftlöcher ist zu verzichten, denn diese wirken dem Isoliereffekt entgegen ( $\triangleright$ Abb. 1).

Die Patienten werden behutsam mit einer Hand fixiert ( $\downarrow$ Abb.2). Ist ein Tier unruhig in der Hand, kann die freie Hand vorsichtig dessen Sicht verdunkeln und es wird ruhiger werden.

Zur Geschlechtsbestimmung wird der Schwanz vorsichtig an seinem Ansatz maximal ca. $65^{\circ}$ nach dorsal gebogen und von ventral betrachtet. Werden die beiden durch die Hemipenisse hervorgerufenen Wölbungen kaudal der Kloake sichtbar, handelt es sich um einen Bock. Zwischen diesen Wölbungen liegt eine Vertiefung. Bei einem Weibchen befindet sich eine halbmondförmige Erhebung direkt kaudal der Kloake. Fast immer sind die Femoralporen beim männlichen Tier deutlicher als beim weiblichen ausgebildet. ( $\triangleright$ Abb.3).

Eine perorale Arzneimittelapplikation findet am einfachsten mittels Knopfkanüle statt ( Abb.4). Subkutane Injektionen werden paramedian flach unter die Rückenhaut gegeben.

Nierenschädigende Arzneimittel oder solche, die über die Niere ausgeschieden werden, sollten unter keinen Umständen in den kaudalen Körperteil injiziert werden, denn Bartagamen verfügen - wie alle Reptilien - über ein renales Pfortadersystem.

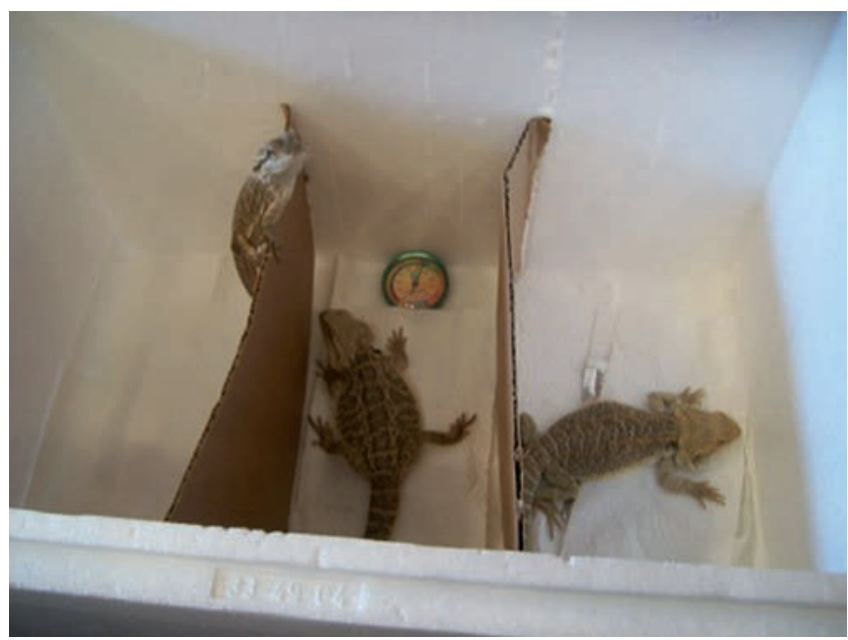

Abb. 1 Der Transport erfolgt in einem thermostabilen und blickdichten Behältnis. Jede Agame wird getrennt transportiert.

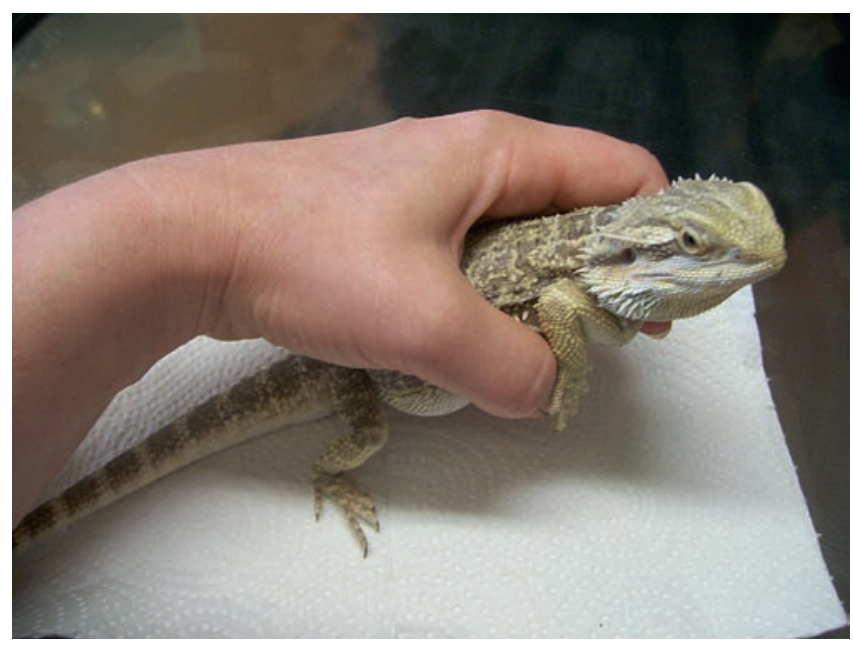

Abb. 2 Handling einer Bartagame. 

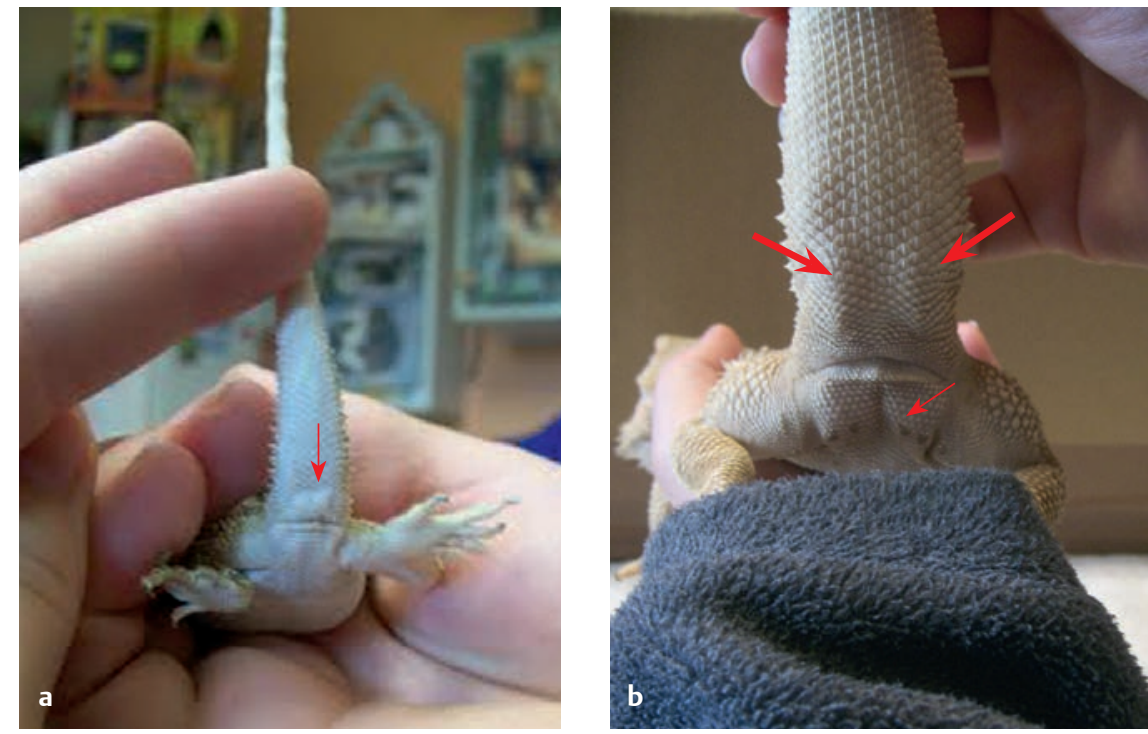

Abb. 3 Geschlechtsbestimmung. a) Weibliches Tier: halbmondförmige Erhebung direkt kaudal der Kloake (Pfeil). b) Männliches Tier: Hemipenisse (große Pfeile) und deutliche Femoralporen (kleiner Pfeil).

Eine intravenöse Medikamentengabe kann über die Schwanzvene erfolgen (z.B. Kalzium bei akuter Tetanie). Hier kann auch Blut entnommen werden.

\section{Krankheitsprophylaxe}

Je langsamer die Tiere wachsen, desto besser ist dies für ihre Gesundheit. Sogenannte Dampfaufzuchten sind abzulehnen.

Wildtiere verbergen Krankheitssymptome so weit als möglich. Ein erster Indikator für ein evtl. pathologisches Geschehen ist eine Gewichtsabnahme. Für den ungeübten Halter, der den Ernährungs- zustand des Tieres evtl. nur schwer beurteilen kann, ist ein regelmäßiges Wiegen der Bartagame sinnvoll ( $\triangleright$ Abb.5). Bei einer Körpermasseabnahme ab 10\% sollte ein Bartagamen kundiger Tierarzt kontaktiert werden.

Neuzugänge sind in einem Quarantänebecken auf Küchenpapier unterzubringen. Ganz wichtig ist es, von jedem Neuzugang während einer mindestens 8-wöchigen Quarantäne zumindest einmal Kot, am besten Sammelkot, parasitologisch untersuchen zu lassen. Hierbei ist das Augenmerk insbesondere auf das Vorkommen von:

- Kokzidien
- Oxyuren

- Kryptosporidien

zu richten. Bei Kryptosporidien ist es wichtig, solche der Futtertiere von denen der Bartagame zu unterscheiden.

Von jedem Tier resp. jeder Gruppe sollte routinemäßig 2-mal jährlich eine Sammelkotprobe parasitologisch untersucht werden, am besten:

- im Frühjahr

- 8 Wochen vor Einleitung der Winterruhe

Für Laboruntersuchungen eignet sich das Labor Exomed (www.exomed.de) besonders, denn es ist auf die Diagnostik der Proben niederer Wirbeltiere und Invertebraten spezialisiert.

\section{Häufige haltungs- und fütterungsbedingte Vorstel- lungsgründe}

Bartagamen werden in der Praxis oft aufgrund haltungs- und fütterungsbedingter Erkrankungen vorgestellt.

\section{Inappetenz und Schwäche}

Ein häufiger Grund für die Konsultation ist Inappetenz, oft in Kombination mit Schwäche. Ohne adäquate Therapie können die Patienten hochgradig abmagern ( Abb.6).

Appetitlosigkeit und Apathie kommen z.B. bei der mangelbedingten Stoffwechselstörung Rachitis vor. Bei der ausgeprägten Form liegen Anzeichen wie

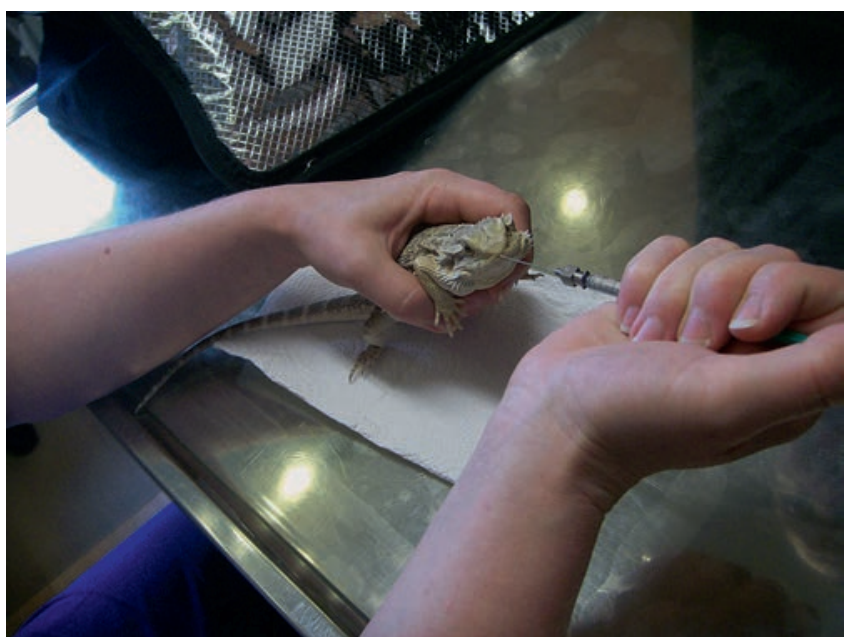

Abb. 4 Perorale Arzneimitteleingabe.

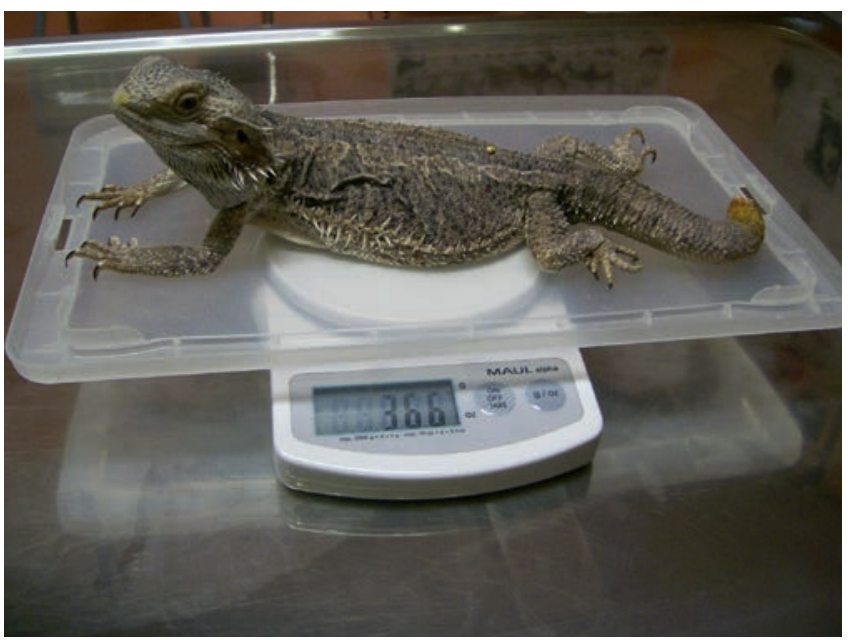

Abb. 5 Ein regelmäßiges Wiegen der Bartagame ist für den unerfahrenen Halter sinnvoll. 

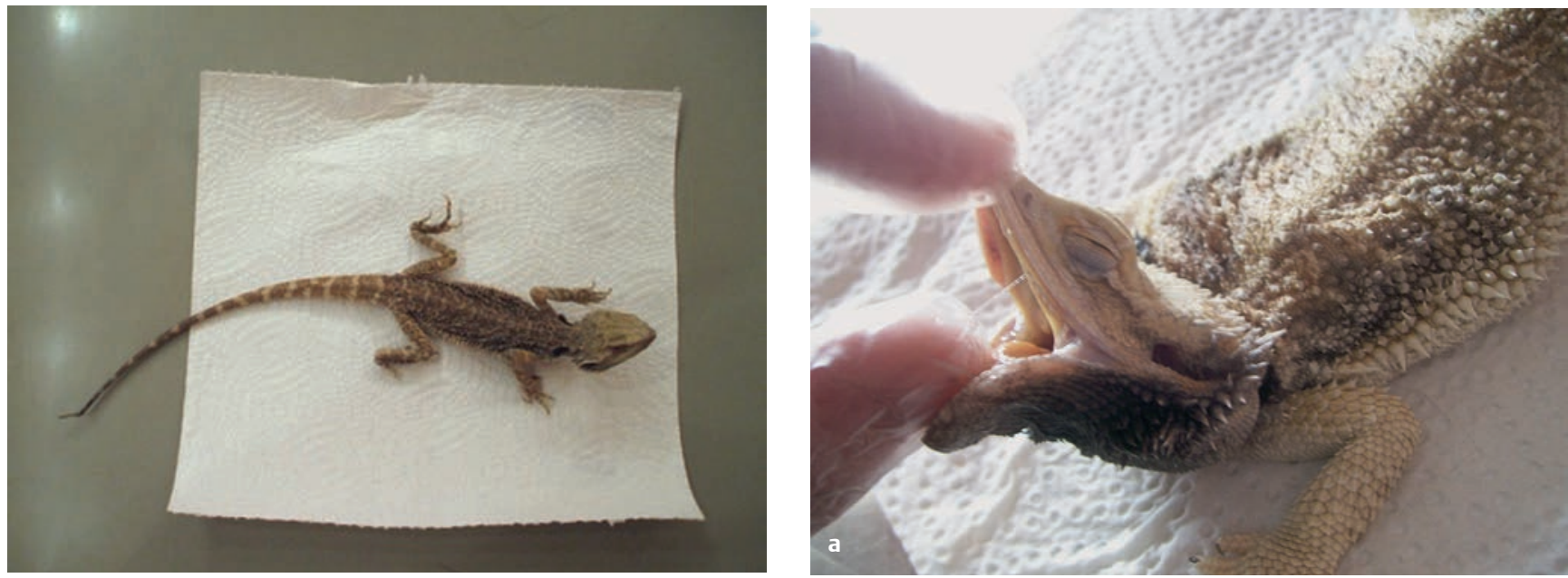

Abb. 6 Hochgradige Abmagerung.

Gummikiefer, Gummischädel und Frakturen vor ( $\mathbf{A b}$ b. 7 ).

Auch Parasitosen des Magen-DarmTraktes wie Oxyuridose, Kokzidiose sowie Kryptosporidiose können zu Inappetenz und Schwäche führen. In der Wildbahn sind die meisten Reptilien von Parasiten befallen ohne zu erkranken. Im Terrarium allerdings können sich Parasiten mit direktem Lebenszyklus explosionsartig vermehren und Parasitosen verursachen. Um dem vorzubeugen, ist Kot nach Möglichkeit schnellstens aus dem Terrarium zu entfernen. Auch sind regelmäßige koproskopische Untersuchungen angezeigt.

Bei Bartagamen - wie bei allen Reptilien - sind antiparasitäre Behandlungen nur nach Diagnostik durchzuführen.

Pressen aufgrund eines durch Parasiten gereizten Darmes kann zu einem Kloakenvorfall führen. Es können aber auch weitere innere Organe wie der Enddarm, der Legedarm, die Hemipenisse oder sogar die Nieren vorfallen ( $\bullet$ Abb. 8 ).

Auch bei einer Legenot zeigen die Agamen Appetitlosigkeit und Schwäche. Insbesondere die psychische Form ist haltungsbedingt.

Bei Infekten der unteren Atemwege, die meist bakteriell bedingt sind und bei suboptimaler Haltung auftreten können, zeigen die Tiere ebenso Inappetenz und Schwäche. Bei Pneumonien sind die
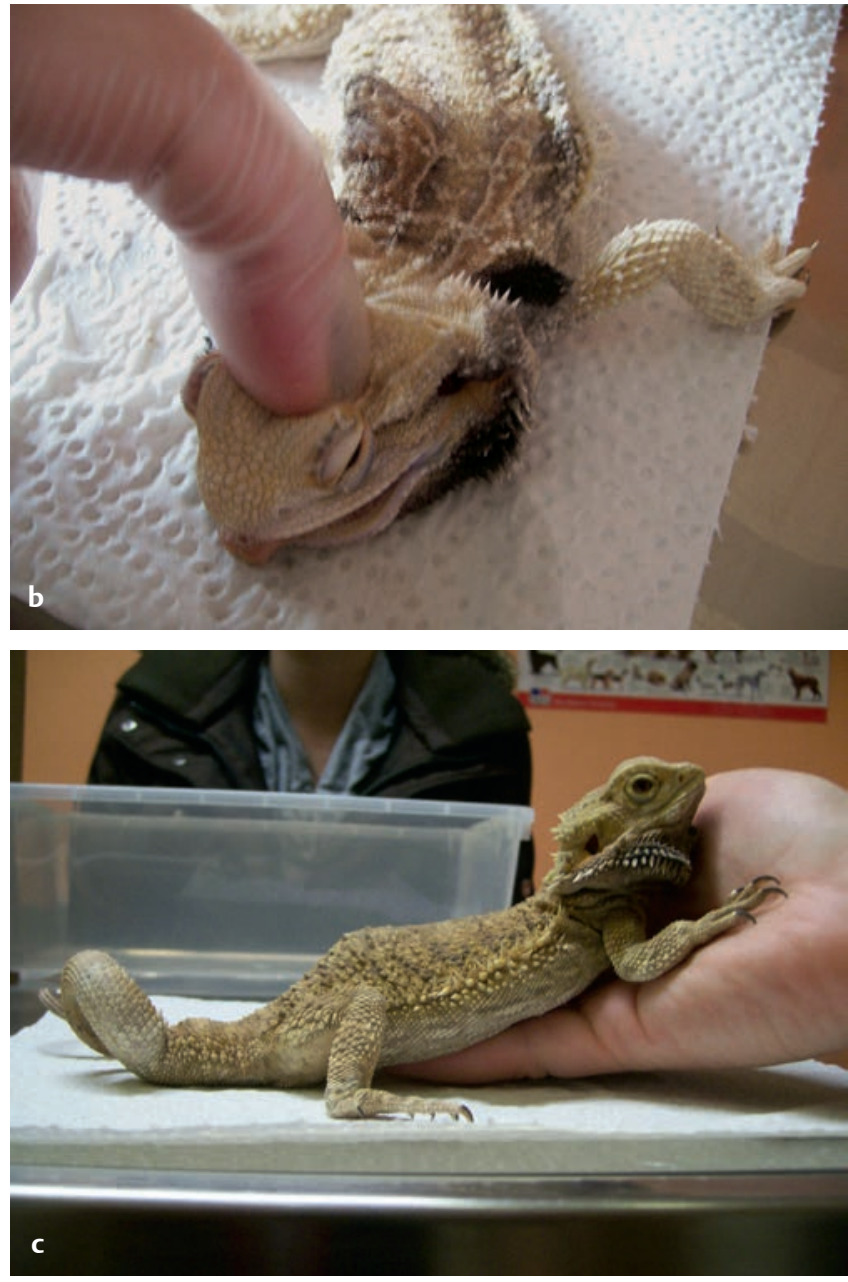

sackförmigen Lungen oft hochgradig mit zähem Schleim gefüllt, der wegen des fehlenden Zwerchfells nicht abgehustet werden kann. Ein Antibiogramm ist zum Erfassen der Resistenzlage zu empfehlen. Insbesondere bei vorliegender Lungenentzündung färben sich Bart und Rückenhaut bei sehr schlechtem Allgemeinzustand schwarz ( Abb. 9).
Ein weiterer Vorstellungsgrund in der Praxis ist Durchfall. Dieser kann parasitär bedingt sein, oftmals ist er aber ernährungsbedingt, wenn den Tieren Obst, Gemüse sowie Salat gefüttert wird. Durch Dehydratation können die Tiere geschwächt sein.

\section{Durchfall und Obstipationen}



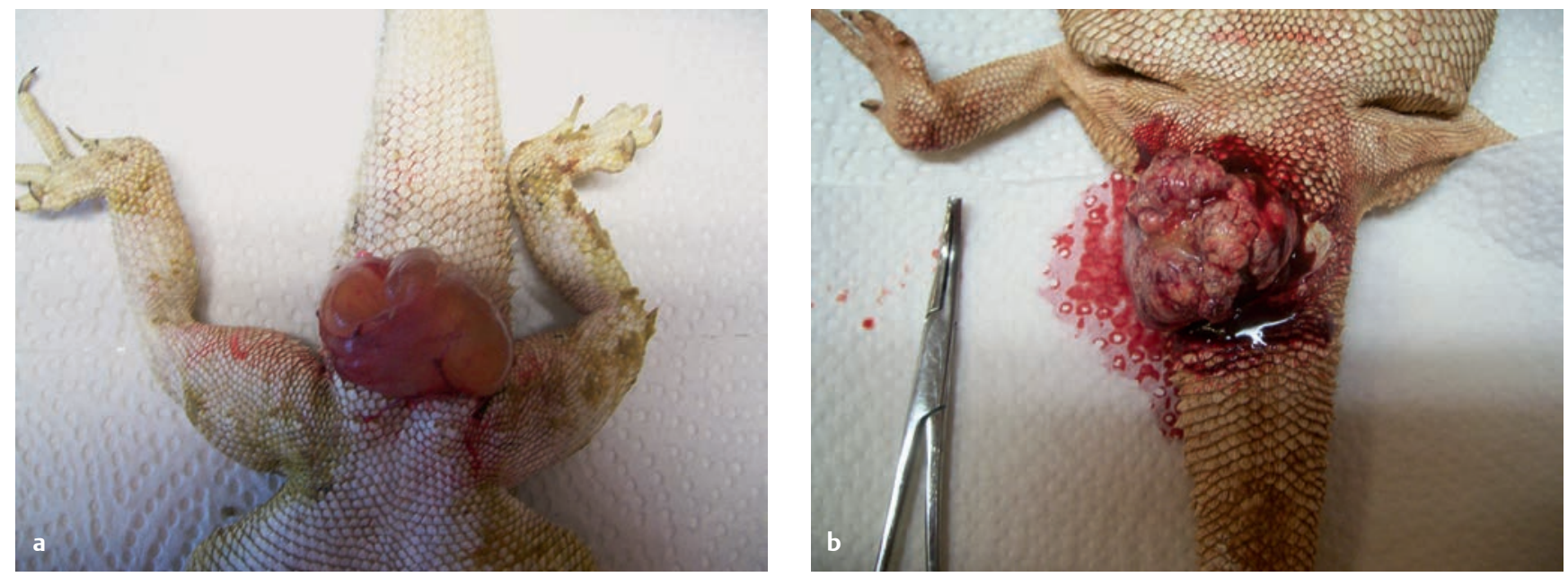

Abb. 8 a) Kloakenprolaps, b) Nierenprolaps.
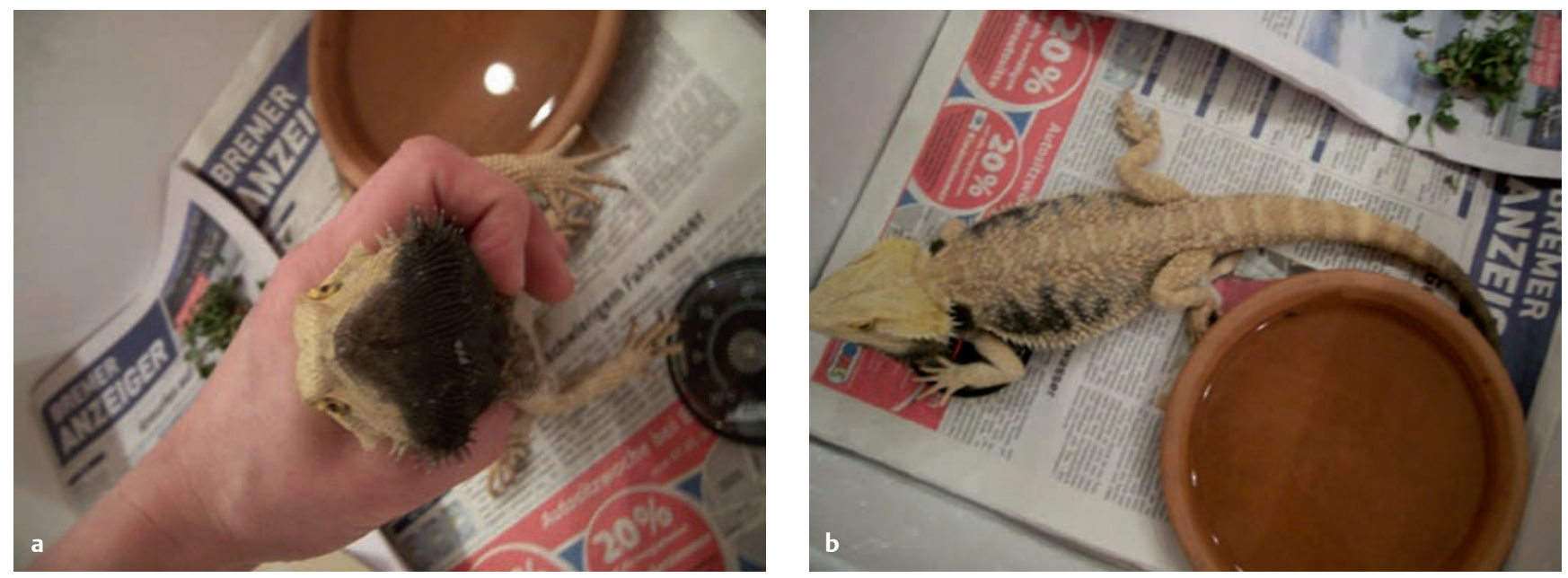

Abb. 9 Pathologische Verfärbungen bei einem Tier mit hochgradiger Pneumonie.
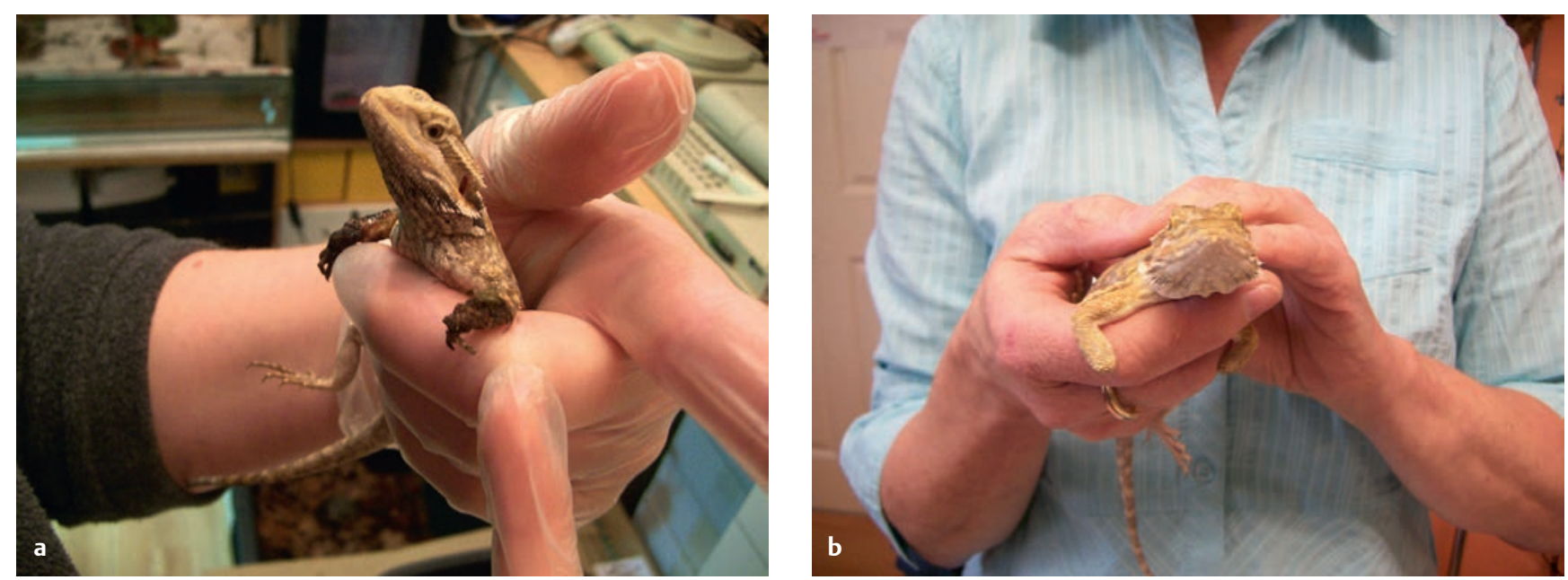

Abb. 10 Nekrose beider Unterarme (a) und verheilte Stümpfe (b). Zwischen den 2 Bildern liegen 18 Wochen. Das Tier wurde antibiotisch und analgetisch versorgt und die nekrotischen Bezirke lokal behandelt. Während der gesamten Zeit war das Tier von gutem Allgemeinbefinden und fraß selbständig. 


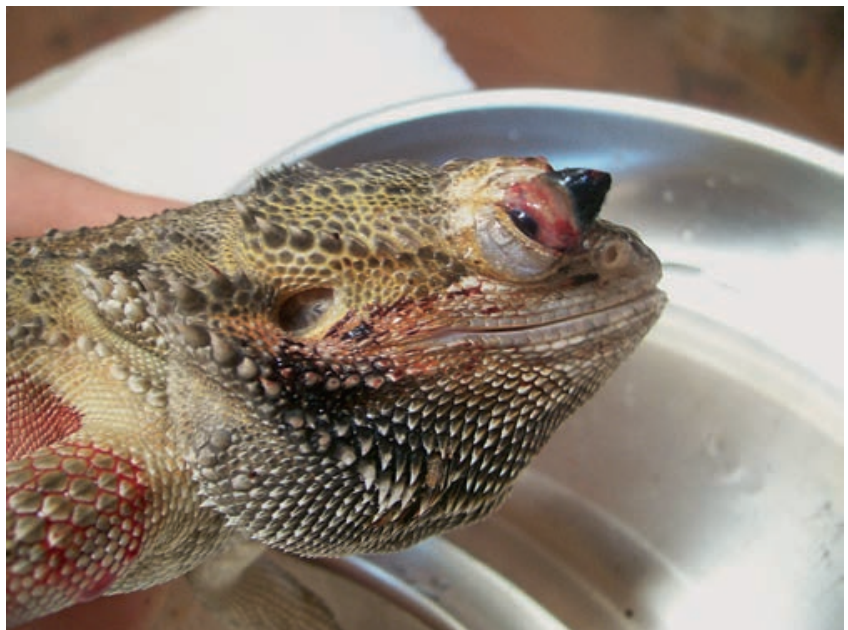

Abb. 11 Bulbusprolaps infolge inadäquater Unterbringung, bei der eine schwere Holzplatte auf das Tier fiel. Das Auge wurde exstirpiert, nachdem der Polytrauma-Patient über 3 Tage stabilisiert worden war. Das Tier kommt jetzt gut einäugig zurecht.

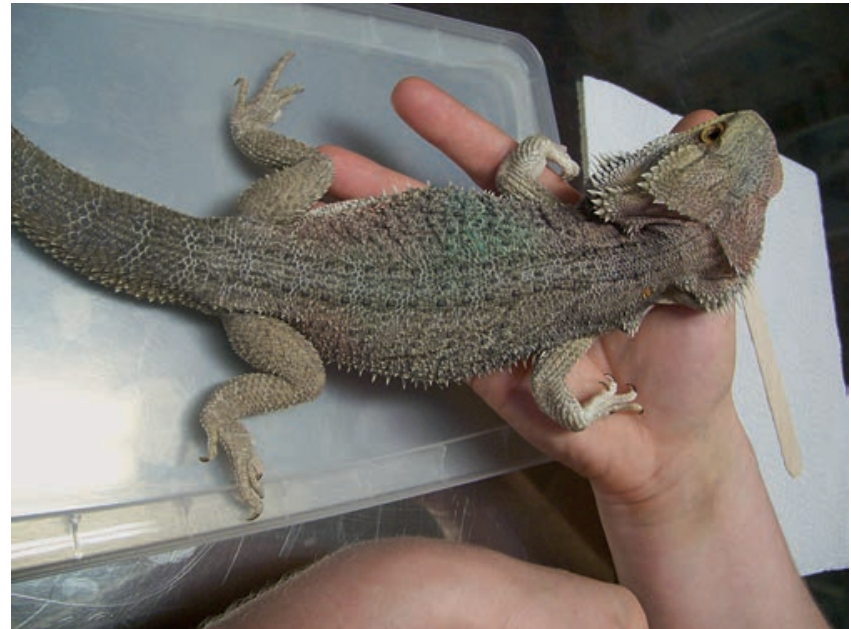

Abb. 12 Grünfärbung: 6 Jahre zuvor wurde das Tier von einem Kind mit grüner Tusche bemalt. Trotz regelmäßiger Häutungen blieb ein grüner Farbstich in der Haut bestehen.
In Ermangelung an Mineralstoffquellen nehmen Bartagamen Sand resp. Steine oral auf. Dies kann zu Anschoppungen im Magen-Darm-Trakt mit Inappetenz und Schwäche führen. Diese Obstipationen müssen aber nicht immer chirurgisch behoben werden.

\section{Verletzungen}

Haltungsbedingte Verletzungen kommen regelmäßig vor und können z.B. durch Bisse von Artgenossen verursacht sein. Schwanzspitzen- und Zehennekrosen entstehen aber nicht nur durch Bisse, sondern treten auch aufgrund oftmals haltungsbedingter inkompletter Häutungen auf, infolge derer die Akren einschnüren und absterben. Die auftretenden nekrotischen Bezirke bedürfen in der Regel keiner Amputation, denn das nekrotische Gewebe kapselt sich ab und löst sich vom gesunden. Die Autorin hat in ihrer Praxis regelmäßig gute Erfahrung mit systemischer analgetischer und

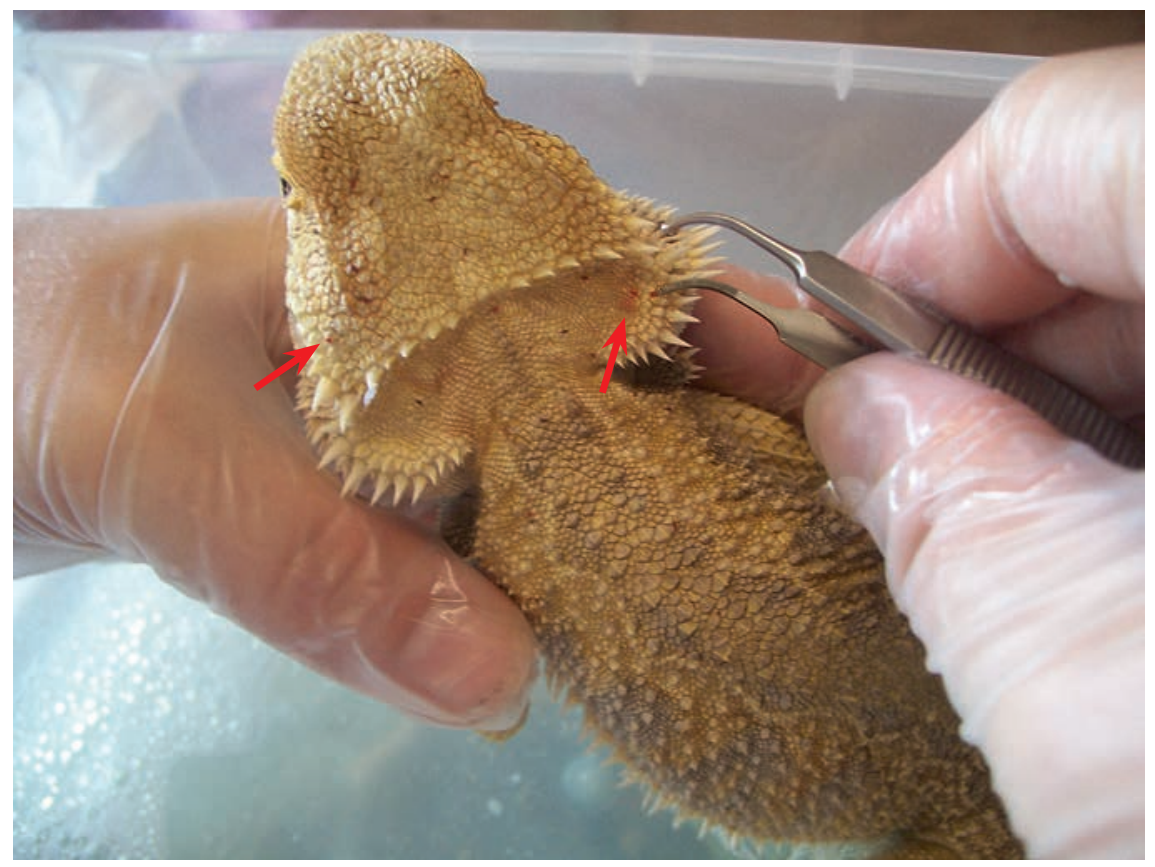

Abb. 13 Blutmilben (Pfeile).

antibiotischer Behandlung sowie gleichzeitiger lokaler Wundversorgung bei solchen Patienten gemacht ( $\triangleright$ Abb.10). Haltungsbedingt werden auch Arm-, Bein- und Wirbelsäulenfrakturen nach Stürzen, z.B. vom Balkon, vorgestellt. Liegt eine Fraktur der Wirbelsäule mit Lähmung der Hinterhand vor, ist die Prognose in vielen Fällen nicht infaust. Wenn die Tiere Harn und Kot absetzen, eingeschränkt in ihrer Bewegung gehalten und mit einer Mischung aus Biose$\operatorname{rin}^{\circledR}$ (WDT), Wasser und Korvimin ${ }^{\circledR}$ VapoVit (WDT) ernährt werden, ist die Lähmung nach 3-6 Monaten oft aufgehoben.

Eine inadäquate Unterbringung kann lebensbedrohliche Folgen haben ( Abb.11). Die Tiere können Verbrennungen erleiden, weil die Lampe im Becken nicht korrekt angebracht ist oder aufgrund eines Zimmerbrandes. Katzen können lebensbedrohliche Bissverletzungen verursachen: Halter mit Katzen sollten Terrarienschlösser zum Schutz der Insassen anbringen. Auch Hautveränderungen aufgrund kindlicher Malereien wurden dokumentiert ( $\triangleright$ Abb. 12).

\section{Andere haltungs- und fütterungs- bedingte Vorstellungsgründe}

Gelegentlich werden in der Praxis Tiere mit Schlundverstopfung durch inadäquates Futter oder mit Darmverschluss durch Fremdkörper vorgestellt. 
Ein häufiger ernährungsbedingter Nebenbefund ist Fettleibigkeit bedingt durch zu reichhaltige Kost.

Auch Hautmilben, die sich insbesondere im Maulwinkel, in Gelenksbeugen, perikloakal sowie -okulär aufhalten, bei starkem Befall aber auch am ganzen Körper zu finden sind, können sich haltungsbedingt stark ausbreiten ( $\triangleright$ Abb. 13). Anstatt mit Chemikalien kann die Umgebung erfolgreich mit Raubmilben, sog. Dutchy's ${ }^{\circledR}$ (www.refona.nl), behandelt werden. Zur Behandlung dieser Lästlinge am Patienten wird entschieden vom Einsatz von Spot-on-Präparaten oder Sprays auf Alkoholbasis abgeraten.

\section{Fazit}

Für die Haltung von Wildtieren in Menschenhand sind Kenntnisse über deren Lebensgewohnheiten sowie über das natürliche Habitat essenziell. Für die optimale Gesundheit einer Bartagame ist ihre artgerechte Haltung und Fütterung im Terrarium ausschlaggebend.

Um Reptilien, wie z.B. Bartagamen, erfolgreich zu therapieren, müssen Art und Geschlecht sicher erkannt werden, Wissen über speziesspezifische Haltungsbedingungen sowie Erfahrung in der Haltung vorhanden sein.

Haltungs- und ernährungsbedingte Erkrankungen kommen häufig in der Reptilienpraxis vor.
Alle Abbildungen: @ K. A. Dörnath

Online zu finden unter

http://dx.doi.org/10.1055/s-0034-1384443

\section{K. Alexandra Dörnath}

MSc Wild Animal Health

Tierarztpraxis Klein Mexiko

Bennigsenstraße 1b

28205 Bremen

info@exotenpraxis-bremen.de

www.exotenpraxis-bremen.de 